\title{
Performance Evaluation and Optimal Management of Distance-Based Registration Using a Semi-Markov Process
}

\author{
Jae Joon Suh, ${ }^{1}$ Kyeongtaek Kim, ${ }^{2}$ Hee-Seon Jang, ${ }^{3}$ and Jang Hyun Baek ${ }^{4}$ \\ ${ }^{1}$ Department of Industrial \& Management Engineering, Hanbat National University, Daejeon 34158, Republic of Korea \\ ${ }^{2}$ Department of Industrial \& Management Engineering, Hannam University, Daejeon 34430, Republic of Korea \\ ${ }^{3}$ Department of Computer, Pyeongtaek University, Pyeongtaek 17869, Republic of Korea \\ ${ }^{4}$ Department of Industrial \& Information Systems Engineering, Chonbuk National University, Jeonju 54896, Republic of Korea
}

Correspondence should be addressed to Jang Hyun Baek; jbaek@jbnu.ac.kr

Received 23 January 2017; Accepted 27 March 2017; Published 10 May 2017

Academic Editor: Azah Mohamed

Copyright (C) 2017 Jae Joon Suh et al. This is an open access article distributed under the Creative Commons Attribution License, which permits unrestricted use, distribution, and reproduction in any medium, provided the original work is properly cited.

\begin{abstract}
We consider the distance-based registration (DBR) which is a kind of dynamic location registration scheme in a mobile communication network. In the DBR, the location of a mobile station (MS) is updated when it enters a base station more than or equal to a specified distance away from the base station where the location registration for the MS was done last. In this study, we first investigate the existing performance-evaluation methods on the DBR with implicit registration (DBIR) presented to improve the performance of the DBR and point out some problems of the evaluation methods. We propose a new performance-evaluation method for the DBIR scheme using a semi-Markov process (SMP) which can resolve the controversial issues of the existing methods. The numerical results obtained with the proposed SMP model are compared with those from previous models. It is shown that the SMP model should be considered to get an accurate performance of the DBIR scheme.
\end{abstract}

\section{Introduction}

In a mobile communication system, continuous management of the mobile station (MS) location is required to connect an incoming call to the user, since the user is continually moving. A series of processes to update user-location information in the mobile communication system database is termed location registration (LR).

Various LR schemes have been proposed for mobile communication systems [1-8]. They include a DBR scheme [1-5], a movement-based registration (MBR) scheme $[2,6,7]$, and a zone-based registration (ZBR) scheme [8]. A mobile communication system adopts an LR scheme considering signaling traffic on a wireless channel, ease of implementation, quality of service, maintenance and administration, adaptability to system expansion, and so on.

We consider the DBR scheme. In the DBR scheme, the location of a mobile station (MS) is updated when the MS enters a base station more than a specified distance away from the base station where the location registration of the MS was done last. The DBR scheme has significant merits in that LR happens infrequently compared with the MBR scheme and there is no ping-pong effect, which happens in the ZBR scheme. Hence, various studies on the performanceevaluation methods for the DBR scheme and performance comparison with other LR schemes have been conducted [2].

To improve the performance of the DBR scheme, the DBR considering the implicit registration effect of incoming and outgoing calls, called the DBIR scheme, was proposed and its performance was analyzed by a continuous-time Markov chain (CTMC) or an imbedded Markov chain (IMMC) [3, 5]. In this study, we point out the issues of the existing CTMC and IMMC methods and propose a new analytical model using the semi-Markov process (SMP) in order to address their flaws. Finally, we show the exact performanceevaluation results and the optimal management plan for the DBR scheme using the proposed SMP method in various operational environments. 


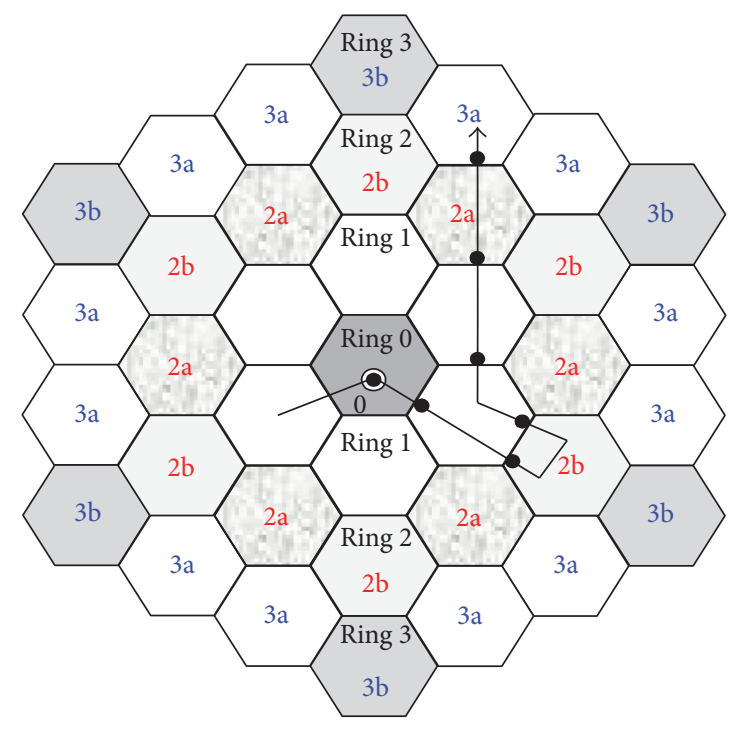

FIGURE 1: Location registration area and ring $(D=4)$ in hexagonal cell structure.

\section{Performance-Evaluation Issues of the DBIR Scheme}

2.1. DBR and DBIR Scheme. In the DBR scheme, an MS requests $L R$ to the base station (BS) which it enters when the distance between the current BS and the BS for which it was last registered reaches or exceeds a specified distance threshold, $D$. The region which a BS controls is called a cell, and all the locations of BS staying in the same cell are regarded as being the same as the location of the BS, which is expressed as the latitude and longitude $(X, Y)$. An MS stores the latitude and longitude $\left(X_{r}, Y_{r}\right)$ of the BS for which it was last registered and receives the new latitude and longitude $\left(X_{c}, Y_{c}\right)$ of the BS whose cell it enters. The moving distance of the MS (DISTANCE) is calculated as follows [1]:

$$
\text { DISTANCE }=\left\lfloor\frac{\sqrt{(\Delta \text { lat })^{2}+(\Delta \text { long })^{2}}}{16}\right\rfloor,
$$

where $\Delta$ lat $=X_{c}-X_{r}$ and $\Delta$ long $=\left(Y_{c}-Y_{r}\right) \times \cos (\pi / 180 \times$ $\left.X_{r} / 14400\right)$. The above equation reflects the curvature of the Earth's surface. The DISTANCE is defined as the minimum number of cells of convenience in this study [2-5]. In Figure 1, for example, the DISTANCE between two neighboring cells is 1 and the DISTANCE between a ring 0 cell and one of the ring 2 cells is 2 .

In the hexagonal configuration, Figure 1 shows the location area of an MS when the MS registers its location in the ring 0 cell and the distance threshold, $D=4$. In the case of the general distance threshold $D$, the location area is composed of $D$ rings, that is, ring 1 , ring $2, \ldots$, ring $(D-1)$ with ring 0 as the center.

According to the CDMA recommendation [1], when an MS sends an Origination Message or Page Response Message successfully, the BS can trace the MS's location. This process is called an implicit registration. Namely, when an outgoing call from an MS or an incoming call to an MS occurs successfully, the BS can perceive the cell to which the MS belongs from the Origination Message or the Page Response Message without another location registration message. Therefore, if a mobile cellular network adopts the DBR scheme with implicit registration, the number of LR can be reduced in the network and the DBR performance in the network can be improved using the implicit registration. The performance of implicit registration will be improved as incoming or outgoing calls increase. This DBR scheme with implicit registration is called $\operatorname{DBIR}[3,5]$.

Now in the next sections, we investigate the existing performance-evaluation methods for the DBIR scheme and point out mistakes in their modeling.

\subsection{Existing Performance-Evaluation Methods for the DBIR Scheme}

Assumptions. Supposing the mobile communication network is configured of hexagonal cells with the same size as shown in Figure 1, the basic assumptions to analyze the performance are as follows:

(i) When an MS leaves a cell, the probability that the MS will enter one of the six neighboring cells is equal to $1 / 6$ for each cell.

(ii) The cell sojourn time $T_{m}$ follows a general distribution with a mean of $1 / \lambda_{m}$.

(iii) The interarrival time $T_{i c}$ of an incoming call to an MS and the interarrival time $T_{o c}$ of an outgoing call from an MS are exponentially distributed, with a mean of $1 / \lambda_{i c}$ and a mean of $1 / \lambda_{o c}$, respectively. That is, the integrated call generating time $T_{c}$ of an MS considering incoming and outgoing call is exponentially distributed with a mean $1 / \lambda_{c}\left(=1 /\left(\lambda_{i c}+\right.\right.$ $\left.\lambda_{o c}\right)$ ).

2.2.1. CTMC Model. The performance-evaluation method for the DBIR scheme using CTMC was proposed in [3] and the registration cost was evaluated. To estimate the registration cost in the DBIR scheme using CTMC, a simple random-walk model was derived considering the mobility characteristics of MS under the above assumptions, as shown in Figure 2.

In Figure 2, state $i$ means that the MS resides in a ring $i$ cell. In this case, for example, an MS in ring 2 cell enters (i) a ring 1 cell with transition rate $\lambda_{m} / 4$, (ii) a ring 2 cell with transition rate $\lambda_{m} / 3$, (iii) a ring 3 cell with transition rate $5 \lambda_{m} / 12$, or (iv) the ring 0 cell with transition rate $\left(\lambda_{i c}+\lambda_{o c}\right)$ when an incoming or outgoing call is generated. With the $D \times D$ transition rate matrix $Q$ corresponding to the above transition diagram, the steady-state probability $\pi_{i}$ of state $i$ can be calculated by the following balance equations [9]:

$$
\begin{aligned}
\Pi \cdot Q & =0, \\
\sum_{i} \pi_{i} & =1,
\end{aligned}
$$




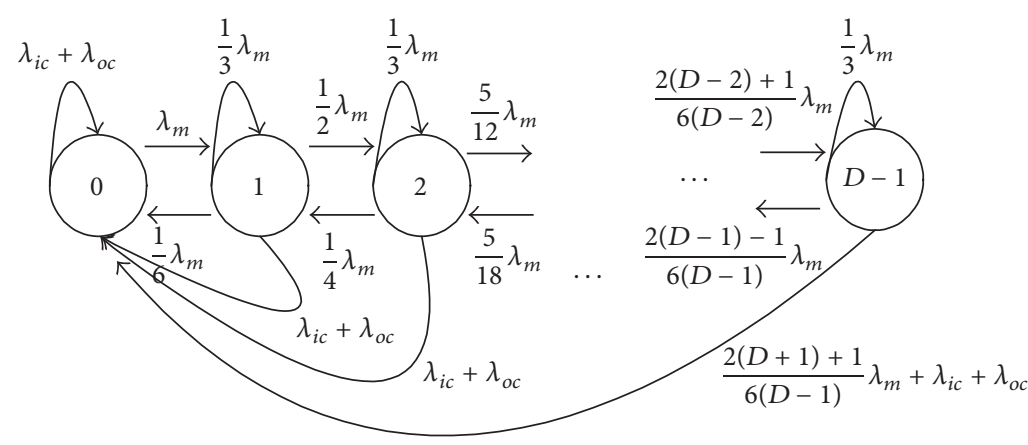

FIGURE 2: State-transition diagram of the DBIR scheme using CTMC.

where $\Pi$ is $\left\{\pi_{0}, \pi_{1}, \ldots, \pi_{D-1}\right\}$. Finally, the registration cost of DBIR using CTMC, $C_{U}^{\text {dbir }}$, was derived by the following equation [3]:

$$
C_{U}^{\mathrm{dbir}}=U \pi_{D-1} q_{D-1,0}=U \frac{2(D-1)+1}{6(D-1)} \lambda_{m} \pi_{D-1},
$$

where $U$ denotes the unit registration cost required for one registration and $q_{D-1,0}$ the transition rate from ring $(D-1)$ cell to ring 0 cell.

By the way, the performance-evaluation method for the DBIR scheme using CTMC described until now has some controversial issues as follows.

First, since the CTMC model essentially assumes that the residence time in a state is exponentially distributed, it does not provide an exact solution for other distributions of the residence time.

Second, even in the case of an exponential distribution of the residence time, the CTMC model cannot reflect the self-loop, that is, a transition from state $i$ back to state $i$ [9]. Therefore, if a self-loop exists, as in the case of Figure 2, the CTMC model also does not provide an exact solution.

2.2.2. IMMC Model. To improve the flaws of the CTMC model, an IMMC model was proposed [5]. Figure 3 shows the state-transition diagram of the DBIR scheme using IMMC. In Figure 3, for example, an MS in ring 2 cell enters (i) a ring 1 cell with transition probability $P\left[T_{c}>T_{m}\right] / 4$, (ii) a ring 2 cell with transition probability $P\left[T_{c}>T_{m}\right] / 3$, (iii) a ring 3 cell with transition probability $5 P\left[T_{c}>T_{m}\right] / 12$, or (iv) the ring 0 cell with transition probability $\left(1-P\left[T_{c}>T_{m}\right]\right)$ when an incoming or outgoing call is generated.

With the $D \times D$ transition probability matrix $P$ corresponding to the above transition diagram, the steady-state probability $\pi_{i}$ of state $i$ can be calculated by the following balance equation [9]:

$$
\begin{aligned}
\Pi \cdot P & =1, \\
\sum_{i} \pi_{i} & =1 .
\end{aligned}
$$

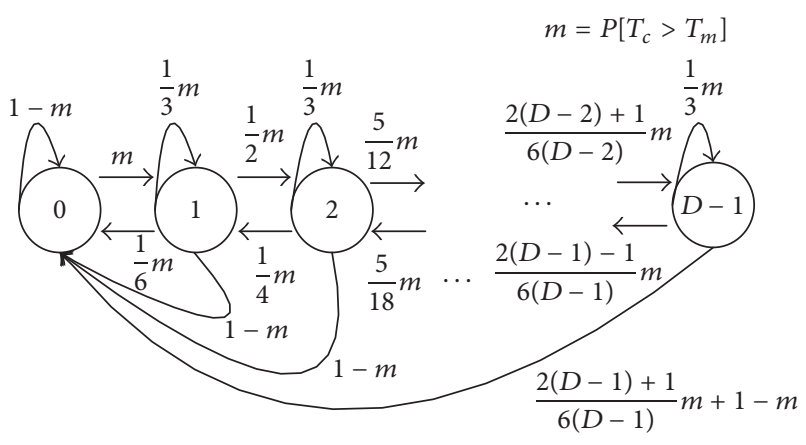

FIGURE 3: State-transition diagram of the DBIR scheme using IMMC.

In the same manner as above, the registration cost of DBIR using IMMC, $C_{U}^{\mathrm{dbir}}$, is derived by the following equation [5]:

$$
C_{U}^{\mathrm{dbir}}=U \frac{2(D-1)+1}{6(D-1)} \lambda_{m} \pi_{D-1}
$$

Unlike in the CTMC model, the IMMC model can reflect the general distribution of cell residence time and the selfloop and thus can obtain a more exact solution than the CTMC model can. However, the IMMC model has still some flaws.

First, let us examine the state 0 (ring 0). In Figure 3, state 0 contains two cases: (i) the case of transition to state 0 when an incoming or outgoing call to/from the MS is generated and (ii) the case of transition to state 0 when the MS in a ring 1 cell enters the ring 0 cell, or the MS in a ring $(D-1)$ cell enters a cell exceeding the distance threshold $D$. However, these two cases should be defined by two separate states, since the residual sojourn time of the state in each case is different.

Second, in the hexagonal cell configuration, the ring $i$ is composed of $6 \times i$ cells, and the transition probabilities to a certain state are not always equal to each other for a certain ring. For example, let us look into the twelve cells belonging to ring 2. As shown in Figure 1, six ring 2 cells are adjacent to one ring 1 cell, and another six ring 2 cells are adjacent to two ring 1 cells. Hence the transition probabilities from a ring 2 cell to a ring 1 cell are different from each other according 


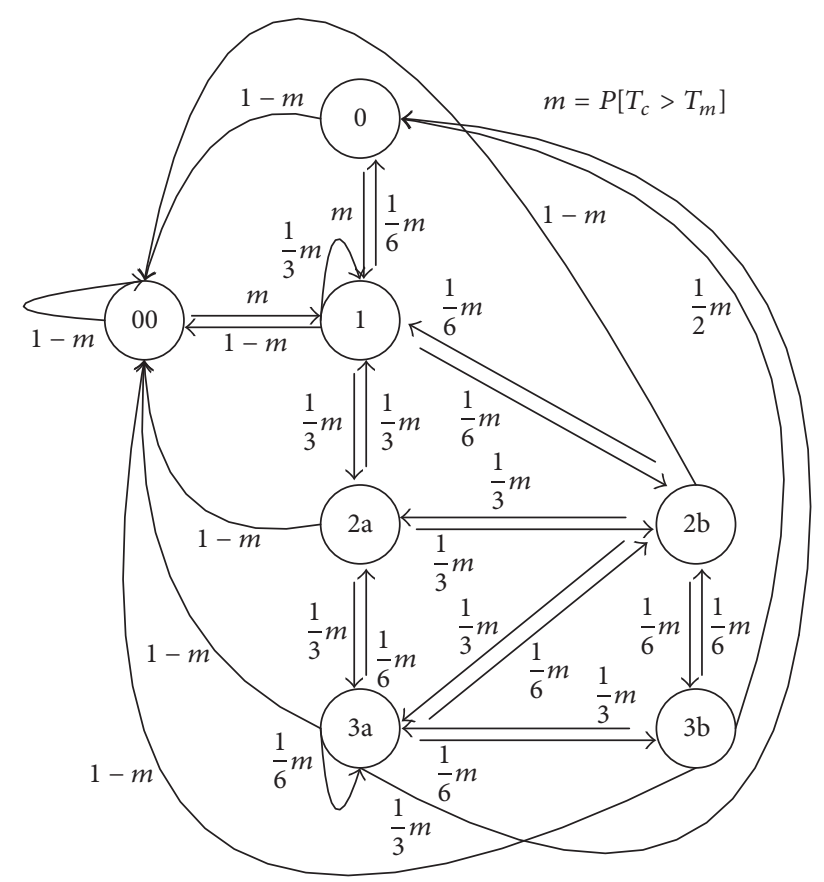

FIGURE 4: State-transition diagram of the DBIR scheme using SMP model.

to the number of adjacent cells, and thus two states should be separately defined with different transition probabilities.

\section{New Performance-Evaluation Method Using SMP}

We propose a new performance-evaluation method using the SMP which can resolve the controversial issues of the existing methods described in Section 2.2. We use the same notations described in Section 2 if they are not defined specifically here. Figure 4 shows the state-transition diagram of the DBIR scheme using SMP for the distance threshold $D=4$. In this SMP model, the cells in a ring greater than ring 1 may be split into two or three states according to the transition probabilities of the cell in the ring. The statetransition diagram for the general distance threshold $D$ is difficult to draw with generalized transition probabilities, but if the distance threshold $D$ is specified, the state-transition diagram could be easily drawn. So we present the statetransition diagram for the distance threshold $D=4$, for convenience.

In Figure 4, state 00 indicates that the present cell of an MS becomes the ring 0 cell because of an incoming or outgoing call, and state 0 indicates that the cell an MS enters becomes the ring 0 cell. That is, the transition to state 0 from any other state happens (1) when an MS in a ring 1 cell enters the ring 0 cell or $(2)$ when an MS in a ring $(D-1)$ cell enters a cell exceeding the distance threshold $D$ and an LR happens. State 1 occurs when an MS stays in a ring 0 cell as shown in Figures 2 and 3 . The ring 2 cells are classified as two states, state $2 \mathrm{a}$ and state $2 b$, which indicate that an MS stays in a cell marked by $2 \mathrm{a}$ or $2 \mathrm{~b}$ in the ring 2 cells of Figure 1 . Similarly, state $3 \mathrm{a}$ or state $3 \mathrm{~b}$ indicates that an MS stays in a cell among the six ring 3 cells marked by $3 \mathrm{a}$ or in a cell among the twelve ring 3 cells marked by $3 \mathrm{~b}$ in Figure 1.

In Figure 4, for example, an MS in state 2a transits to (i) state 1 with transition probability $P\left[T_{c}>T_{m}\right] / 6$, (ii) state 2b with transition probability $P\left[T_{c}>T_{m}\right] / 3$, (iii) state $3 \mathrm{a}$ with transition probability $P\left[T_{c}>T_{m}\right] / 6$, (iv) state $3 \mathrm{~b}$ with transition probability $P\left[T_{c}>T_{m}\right] / 3$, or (v) state 00 with transition probability $\left(1-P\left[T_{c}>T_{m}\right]\right)$ when an incoming or outgoing call is generated. Let us pay attention to the cell sojourn time in state 00 . Since state 00 is a state in which the present cell of an MS becomes the ring 0 cell because of an incoming or outgoing call, the cell sojourn time in state 00 is the residual cell sojourn time for which the MS stays in the present cell; let us denote it by $R_{m}$. Then the transition probability from state 00 to state 1 is expressed by $\left[T_{c}>R_{m}\right.$ ], which can be derived as follows:

$$
\begin{aligned}
P\left[T_{c}>R_{m}\right] & =\int_{0}^{\infty} \int_{r_{m}}^{\infty} \lambda_{c} e^{-\lambda_{c} t_{c}} f_{r}\left(r_{m}\right) d t_{c} d r_{m} \\
& =\frac{1-f_{m}^{*}\left(\lambda_{c}\right)}{\rho},
\end{aligned}
$$

where $\rho=\lambda_{\mathrm{c}} / \lambda_{m}$.

In addition, the transition probability from state 00 to state 00 itself is expressed by $P\left[T_{c}<R_{m}\right]=1-P\left[T_{c}>\right.$ $\left.R_{m}\right]=1-\left(1-f_{m}^{*}\left(\lambda_{c}\right)\right) / \rho$, which means the probability that an incoming or outgoing call to/from the MS is generated before the MS enters another cell.

The $7 \times 7$ transition probability matrix corresponding to the above state-transition diagram is as follows:

$$
P=\left[\begin{array}{cccccc}
1-m & 0 & m & \cdots & 0 & 0 \\
1-m & 0 & m & \cdots & 0 & 0 \\
1-m & \frac{1}{6} m & \frac{1}{3} m & \cdots & 0 & 0 \\
\vdots & \vdots & \vdots & \ddots & \vdots & \vdots \\
1-m & \frac{1}{3} m & 0 & \cdots & \frac{1}{6} m & \frac{1}{3} m \\
1-m & \frac{1}{2} m & 0 & \cdots & \frac{1}{3} m & 0
\end{array}\right] .
$$

Eventually, if the sojourn time in a state is different, the steady-state probability $\widetilde{P}_{i}$ of state $i$ considering the sojourn time in the state can be obtained by means of e (8) after calculating $\pi_{i}$ using $\pi \cdot P=\pi$ and $\sum_{i} \pi_{i}=1$ [9].

$$
\widetilde{P}_{i}=\frac{\pi_{i} \tau_{i}}{\sum_{j} \pi_{j} \tau_{j}},
$$

where $\tau_{i}$ is the mean sojourn time of state $i$ and can be derived as follows:

$$
\begin{aligned}
\tau_{00}= & E\left[T_{c} \mid T_{c}<R_{m}\right] P\left[T_{c}<R_{m}\right] \\
& +E\left[R_{m} \mid T_{c}>R_{m}\right] P\left[T_{c}>R_{m}\right] \\
= & \int_{0}^{\infty} \int_{0}^{r_{m}} t_{c} f_{c}\left(t_{c}\right) f_{r}\left(r_{m}\right) d t_{c} d r_{m} \\
& +\int_{0}^{\infty} \int_{0}^{t_{c}} r_{m} f_{r}\left(r_{m}\right) f_{c}\left(t_{c}\right) d r_{m} d t_{c}
\end{aligned}
$$




$$
\begin{aligned}
\tau_{0}= & E\left[T_{c} \mid T_{c}<T_{m}\right] P\left[T_{c}<T_{m}\right] \\
& +E\left[T_{m} \mid T_{c}>T_{m}\right] P\left[T_{c}>T_{m}\right] \\
= & \int_{0}^{\infty} \int_{0}^{t_{m}} t_{c} f_{c}\left(t_{c}\right) f_{m}\left(t_{m}\right) d t_{c} d t_{m} \\
& +\int_{0}^{\infty} \int_{0}^{t_{c}} t_{m} f_{m}\left(t_{m}\right) f_{c}\left(t_{c}\right) d t_{m} d t_{c}=\tau_{1}=\tau_{2 \mathrm{a}} \\
= & \tau_{2 \mathrm{~b}}=\tau_{3 \mathrm{a}}=\tau_{3 \mathrm{~b}} .
\end{aligned}
$$

Finally, the registration cost of DBIR using SMP, $C_{U}^{\mathrm{dbir}}$, can be derived by

$$
C_{U}^{\mathrm{dbir}}=U \frac{2(D-1)+1}{6(D-1)} \lambda_{m} \widetilde{P}_{D-1} .
$$

Most mobile communication systems adopt simultaneous paging when an incoming call arrives, even if some efficient paging methods have been proposed [2, 4]. For simultaneous paging, the paging cost per unit time, $C_{P}$, is as in

$$
C_{P}=V\left[1+\sum_{i=1}^{D-1} 6 i\right]=V[1+3 D(D-1)],
$$

where $V$ is the unit paging cost for a cell.

The total of the LR cost and the paging cost is as follows:

$$
C_{T}=C_{U}+C_{P}
$$

\section{Numerical Results and Comparison}

To obtain numerical results of the DBIR scheme using the various models described in Sections 2 and 3 and compare them with each other, we assume the following:

$$
\begin{aligned}
U & =1.0, \\
V & =0.1, \\
\lambda_{m} & =1, \\
\lambda_{i c} & =\lambda_{o c}=0.5\left(\lambda_{c}=1\right) .
\end{aligned}
$$

Table 1 shows the LR costs of the various models for various cell sojourn-time distributions. As shown in Table 1, the results for all of the CTMC, IMMC, and SMP models are identical for exponential distribution of cell sojourn time. Thus, let us investigate the LR costs focusing on the cases where the variance of cell sojourn time is greater or smaller than the variance 1 of $\operatorname{Exp}(1)$.

As shown in Table 1, the LR cost of the SMP model is less than that of the IMMC model under the Gamma(1/2, 2) distribution, whereas the LR cost of the SMP model is more than that of the IMMC model under the $\operatorname{Gamma}(2,1 / 2)$ distribution. Note that the LR cost of the SMP model is less or more than that of the IMMC model depending on the cell sojourn-time distributions.
TABLE 1: Registration cost for various cell sojourn time distributions.

\begin{tabular}{lll}
\hline Model and distribution & $D=2$ & $D=3$ \\
\hline CTMC [3] & 0.1875 & 0.0432 \\
IMMC [5] & & \\
Exp(1) & 0.1875 & 0.0432 \\
Gamma(1/2,2) & 0.2084 & 0.0559 \\
Gamma(2,1/2) & 0.1714 & 0.0349 \\
U(0,2) & 0.1678 & 0.0332 \\
SMP & & \\
Exp $(1)$ & 0.1875 & 0.0433 \\
Gamma(1/2,2) & 0.1526 & 0.0391 \\
Gamma(2,1/2) & 0.2143 & 0.0416 \\
$U(0,2)$ & 0.2203 & 0.0415 \\
\hline
\end{tabular}

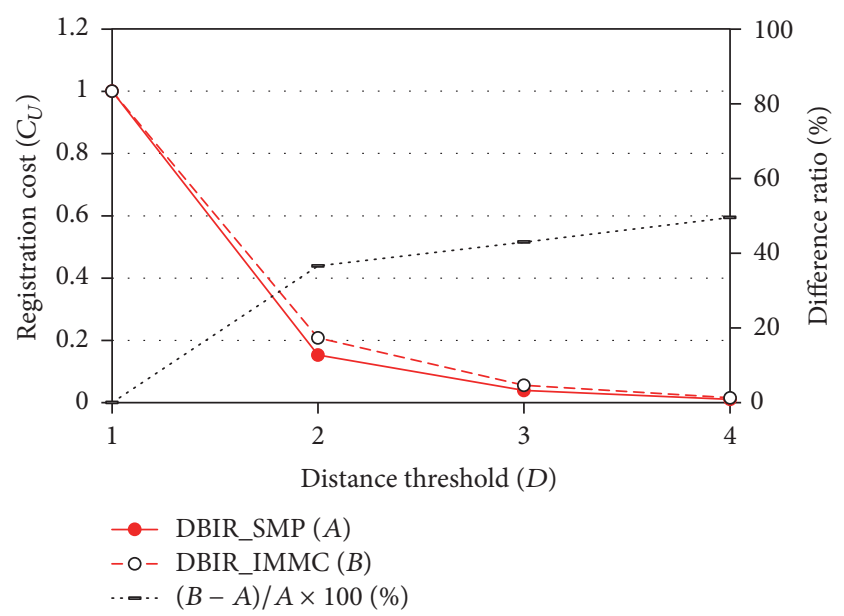

FIGURE 5: Registration cost and its difference ratio $\left(T_{m} \sim\right.$ Gam$\mathrm{ma}(1 / 2,2))$.

According to various distance thresholds under Gamma $(1 / 2,2)$ distribution of cell sojourn time, the exact LR costs using the IMMC and SMP models are shown in Table 2 and Figure 5.

In this case, the LR cost of the IMMC model is more than that of the SMP model by about 37 50\%, reflecting the residual cell sojourn time in the SMP model. In the case of $\operatorname{Gamma}(1 / 2,2)$ distribution, since the expected residual sojourn time $E\left(R_{m}\right)$ in state 00 is greater than $1, \pi_{D-1}$ of the SMP model is smaller than the $\pi_{D-1}$ of the IMMP model and therefore the LR cost of the SMP model decreases compared with that of the IMMC model. That is, since $E\left(R_{m}\right)$ is different from $E\left(T_{m}\right)$ in $\operatorname{Gamma}(1 / 2,2)$ distribution, it is evident that the SMP model presents an exact performance value by classifying the process of an MS transiting to the ring 0 cell into two states (states 0 and 00 ) and by properly estimating the cell sojourn time in each state.

Figure 6 shows the exact total signaling costs of the DBR scheme and the DBIR scheme using the proposed SMP model for various distance thresholds. The LR cost of the DBIR scheme is less than that of the DBR scheme for all distance thresholds and thus it is the same for the total signaling cost. With the parameter values assumed in this numerical example, the optimal distance threshold is estimated by two 
TABLE 2: Registration cost and its difference ratio $\left(T_{m} \sim \operatorname{Gamma}(1 / 2,2)\right)$.

\begin{tabular}{lcccc}
\hline & $D=1$ & $D=2$ & $D=3$ & $D=4$ \\
\hline DBIR_SMP $(A)$ & 1.0000 & 0.1526 & 0.0391 & 0.0105 \\
DBIR_IMMC $(B)$ & 1.0000 & 0.2084 & 0.0559 & 0.0157 \\
$(B-A) / A \times 100(\%)$ & 0 & 36.6 & 43.0 & 49.5 \\
\hline
\end{tabular}

TABLE 3: Registration cost for various variances $\left(D=2, T_{m} \sim \operatorname{Gamma}(\alpha, \beta), E\left(T_{m}\right)=1\right)$.

\begin{tabular}{lcccc}
\hline & Variance & SMP $(A)$ & IMMC $(B)$ & $(B-A) / A \times 100(\%)$ \\
\hline $\operatorname{Gamma}(3,1 / 3)$ & $1 / 3$ & 0.2256 & 0.1646 & -27.0 \\
$\operatorname{Gamma}(2,1 / 2)$ & $1 / 2$ & 0.2143 & 0.1714 & -20.0 \\
$\operatorname{Gamma}(1,1)$ & 1 & 0.1875 & 0.1875 & 0.0 \\
$\operatorname{Gamma}(1 / 2,2)$ & 2 & 0.1526 & 0.2084 & 36.6 \\
$\operatorname{Gamma}(1 / 3,3)$ & 3 & 0.1310 & 0.2218 & 69.3 \\
\hline
\end{tabular}

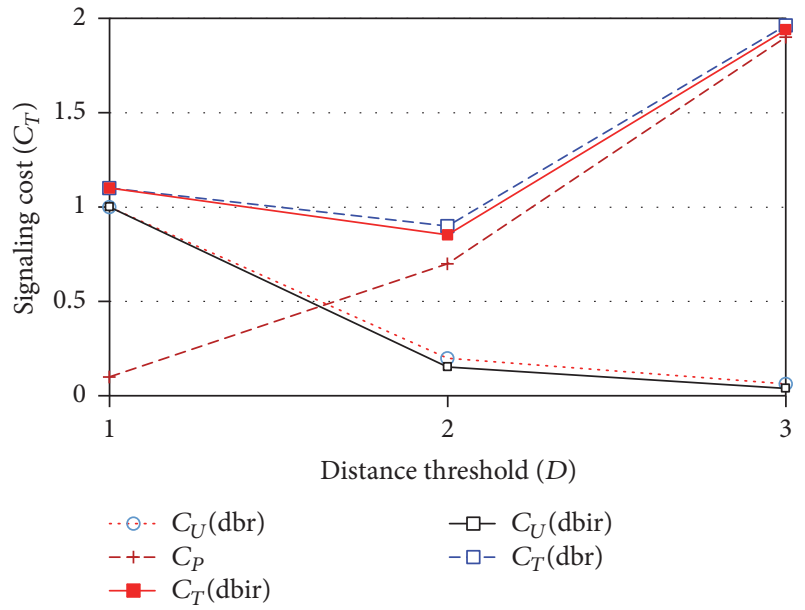

FIGURE 6: Signaling cost versus distance threshold.

as in the case of the CTMC model and the IMMC model. It is identical in the context of the optimal threshold value, but the SMP model should be used in order to exactly analyze the performance improvement effect of the DBIR scheme, especially for general distribution of cell sojourn time.

Shown in Table 3 is the LR cost for various variances of Gamma distribution with the same number of entrances into a cell per unit time. As described above, the IMMC model and the SMP model present an identical LR cost in the case of $\operatorname{Exp}(1)$ distribution of cell sojourn time, because, given the memoryless property of exponential distribution, the mean residual cell sojourn time is the same as the mean cell sojourn time. The SMP model, however, reflects the cell sojourn time of each state in order to exactly analyze the performance of the DBIR scheme.

(1) If the variance of cell sojourn time is greater than the variance of $\operatorname{Exp}(1), \pi_{D-1}$ of the SMP model is smaller than $\pi_{D-1}$ of the IMMP model, since the expected residual sojourn time $E\left(R_{m}\right)$ is greater than 1 in Gamma distribution, and hence the LR cost of the SMP model is less than that of the IMMC model. (2) On the other hand, when the variance of cell sojourn time is less than the variance of $\operatorname{Exp}(1)$, the LR cost of the SMP model is greater than that of IMMP model.
Although only Gamma distribution is presented in Table 3, it can be shown that there is the same tendency for other distributions including uniform distribution considered in Table 1. Consequently, we can notice that the LR cost of the IMMC model is underestimated if the variance of cell sojourn time is small, whereas the LR cost of the IMMC model is overestimated if the variance of cell sojourn time is great. The IMMC model cannot analyze exactly the performance of the DBIR scheme in any case, and therefore the SMP model should be used in order to exactly analyze the performance of the DBIR scheme.

\section{Conclusion}

In this study, we considered distance-based registration (DBR). We pointed out some problems of the previous DBR studies with implicit registration (DBIR) and proposed a new modeling of the DBIR scheme in order to analyze its exact performance. Using the new proposed SMP model, we showed that DBIR is always superior to DBR. In addition, we found that the LR cost of the IMMC model is underestimated if the variance of cell sojourn time is small, whereas the LR cost of the IMMC model is overestimated if the variance of cell sojourn time is great.

To conclude, the DBR scheme should be considered along with implicit registration in order to achieve the best performance, and its performance should be analyzed using the SMP model, especially for general distribution of cell sojourn time.

\section{Conflicts of Interest}

The authors declare that there are no conflicts of interest regarding the publication of this paper.

\section{References}

[1] TIA/EIA/IS-95-B, "MS-BS, compatibility standard for dualmode wideband spread spectrum cellular system," 1999.

[2] Z. Mao and C. Douligeris, "Location-based mobility tracking scheme for PCS networks," Computer Communications, vol. 23, no. 18, pp. 1729-1739, 2000. 
[3] J. H. Baek and B. H. Ryu, "Modeling and analysis of distancebased registration with implicit registration," ETRI Journal, vol. 25, no. 6, pp. 527-530, 2003.

[4] J. Baek, T. Lee, and C. Kim, "Performance analysis of 2location distance-based registration in mobile communication networks," IEICE Transactions on Communications, vol. E96-B, no. 3, pp. 914-917, 2013.

[5] J. Baek, T. Lee, and C. Kim, "Exact modeling and performance analysis of distance-based registration considering the implicit registration effect of outgoing calls," IEICE Transactions on Communications, vol. E95-B, no. 9, pp. 3019-3023, 2012.

[6] J. Li, H. Kameda, and K. Li, "Optimal dynamic mobility management for PCS networks," IEEE/ACM Transactions on Networking, vol. 8, no. 3, pp. 319-327, 2000.

[7] X. Wang, X. Lei, P. Fan, R. Q. Hu, and S.-J. Horng, "Cost analysis of movement-based location management in PCS networks: an embedded Markov chain approach," IEEE Transactions on Vehicular Technology, vol. 63, no. 4, pp. 1886-1902, 2014.

[8] Y. B. Lin, "Reducing location update cost in a PCS network," IEEE/ACM Transactions on Networking, vol. 5, no. 1, pp. 25-33, 1997.

[9] S. Ross, Stochastic Processes, John Wiley \& Sons, 1996. 


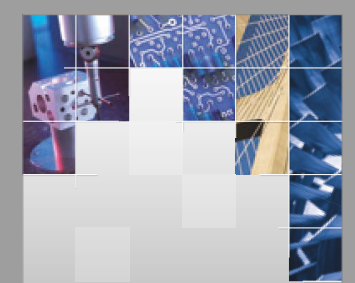

\section{Enfincering}
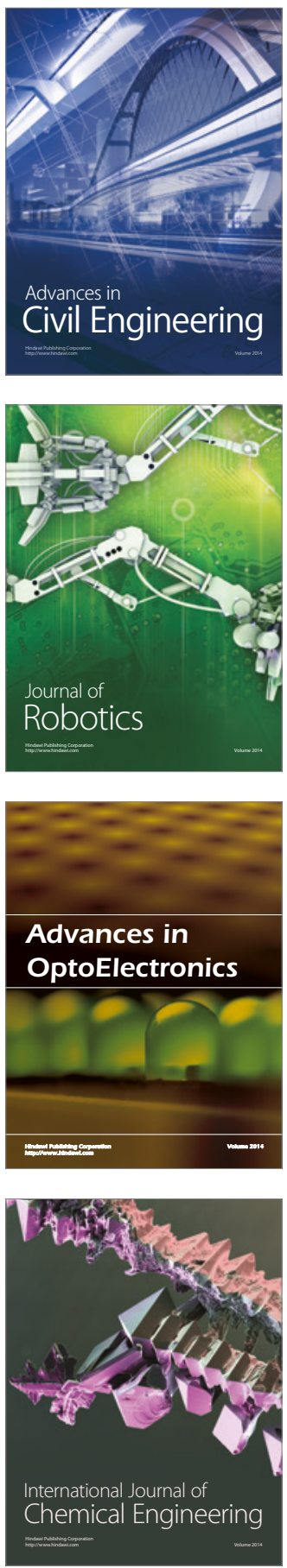

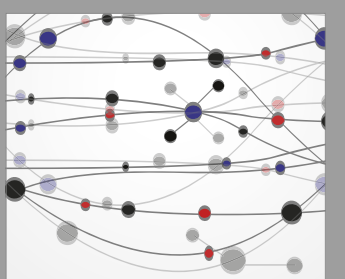

The Scientific World Journal

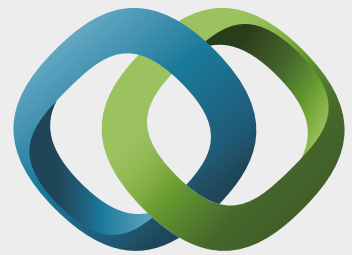

\section{Hindawi}

Submit your manuscripts at

https://www.hindawi.com
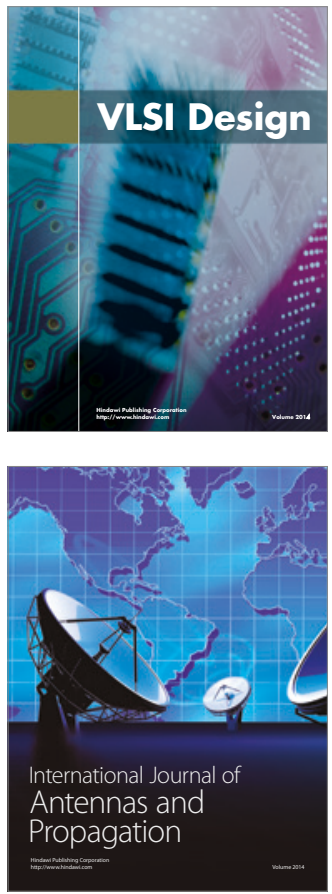

\section{Rotating}

Machinery
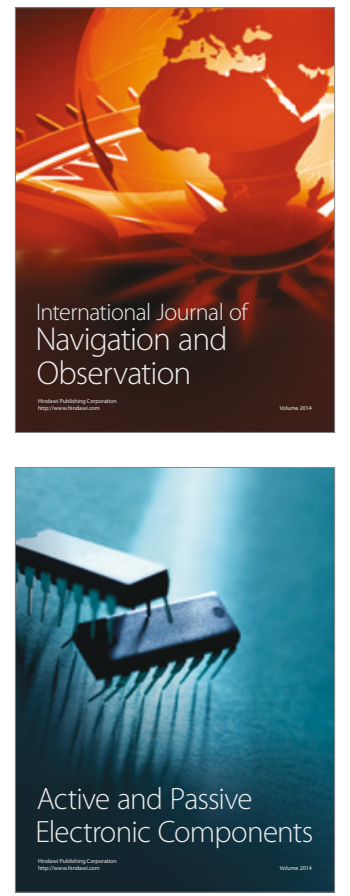
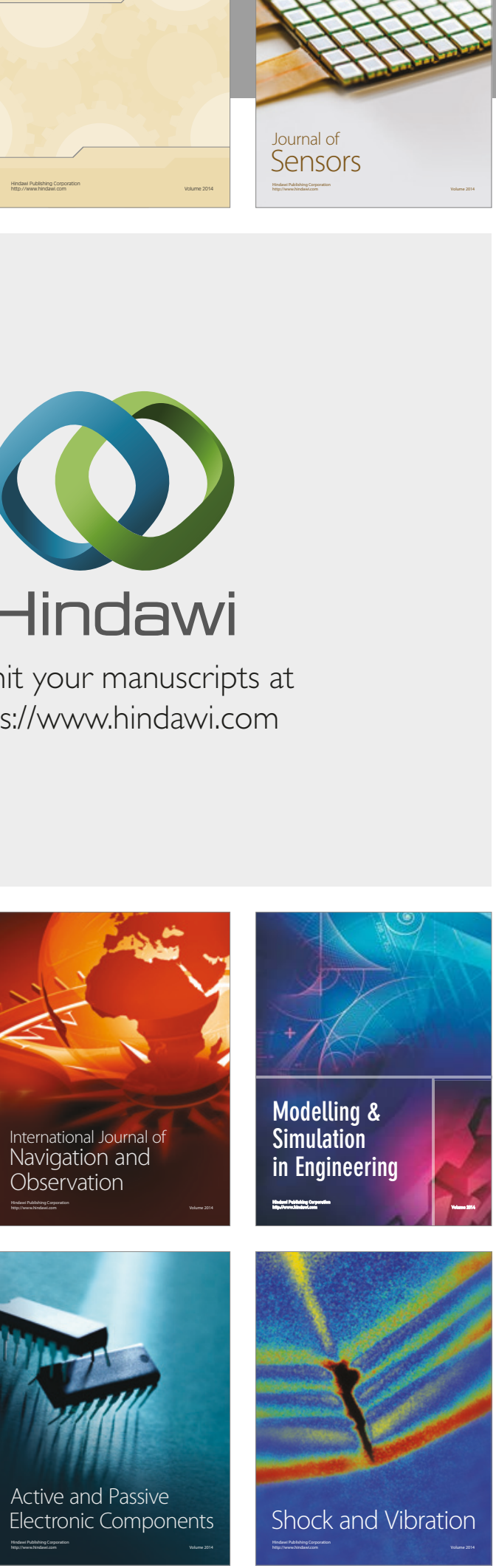
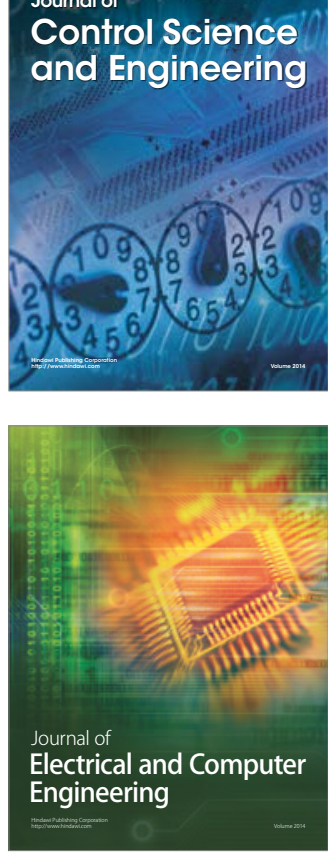

Distributed

Journal of

Control Science

and Engineering
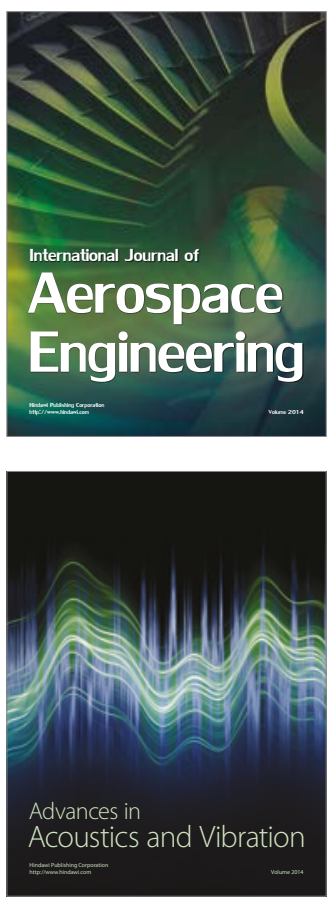

Sensor Networks 